\title{
Role of the Induction Zone on Turbulence-Detonation Interaction
}

\author{
Luca Massa* \& Frank K. Lu ${ }^{\dagger}$ \\ University of Texas at Arlington, Arlington, TX 76019, USA
}

\begin{abstract}
This paper presents an in-depth analysis on the weak turbulence detonation linear interaction problem. The emphasis is on scaling and resonance issues due to the presence of structural scales that are intrinsic to the post-shock heat release region. Natural frequencies are determined as eigenvalues of the non-forced interaction inviscid problem. Influence of detonation parameters on the set of natural frequencies and concomitant effects on the one dimensional power spectra are analyzed. Scaling effects associated with the ratios between longitudinal turbulence length scale and the detonation induction scale are discussed. Acoustic and entropy far-field disturbance patterns are analyzed separately. Analysis of the parameter space reveals a strong link between detonation overdrive and acoustic attenuation. The damping is correlated with the sub-critical nature of the characteristic solutions for high overdrives. High activation energy detonations support resonant interaction with finer turbulence. The range of natural wave numbers increases with the heat release. Entropy patterns of the temperature and density are more strongly affected by resonance than those of the longitudinal velocity. This outcome highlights the thermal nature of the interaction in the induction region. For detonation conditions on the stability boundary, a larger overdrive supports a weaker resonant peak in both the temperature and longitudinal velocity spectra.
\end{abstract}

\section{Introduction}

$\mathrm{W}$ e are interested in the role of the detonation structure on the amplification of turbulence emanating from a detonation wave. The present analysis extends Ribner's work ${ }^{1}$ on shock-turbulence interaction and complements Jackson, et al.'s ${ }^{2,3}$ work on detonation-turbulence interaction by explicitly considering the relation between the longitudinal turbulence length scale and the detonation induction scale, here represented by the half reaction length $l_{1 / 2}$. The incoming turbulence is weak and incompressible; thus, we obtain numerical solutions to the linear interaction problem where the pre-shock turbulence field is obtained as the superposition of planar vorticity waves. The induction region affects the post-shock turbulence field in two basic ways. First, the field is strongly non-homogeneous immediately behind the shock; second, a set of characteristic length scales based on the detonation structure strongly modifies the turbulence intensity at selected frequencies. The first point is addressed by defining an averaging operator in the direction normal to the shock and in time, so that the correlations depend on the distance from the shock. The second point is addressed by determining the eigensolutions to the homogeneous linear interaction where the pre-shock turbulence intensity is set to zero. This paper is organized as follows: the detonation structure equations and the non-dimensionalizing scales are presented in $\S I I$, the pre and post-shock turbulence field are described in $\S I I I$ and $\S I V$, the linearized perturbation equations and the far-field wave decomposition of the perturbation field are detailed in $\S \mathrm{V}$, the homogeneous problem is discussed in $\S \mathrm{VI}$, the numerical method is summarized in $\S$ VII, the main results are discussed in $\S \mathrm{VIII}$ and, finally, the conclusions are given in $\S \mathrm{IX}$.

\footnotetext{
*Assist. Prof., 500 W. First St., WH 214, Arlington, TX, 76019. email: massa@uta.edu. phone: 817-272-0125.

$\dagger$ Professor, Department of Mechanical and Aerospace Engineering, Associate Fellow AIAA.

Copyright (c) 2009 by the American Institute of Aeronautics and Astronautics, Inc. All rights reserved.
} 


\section{Detonation Structure}

The detonation structure is represented using Erpenbeck's model. ${ }^{4}$ We assume a first-order irreversible reaction controlled by an Arrhenius kinetics rate equation,

$$
\tilde{r} \equiv \frac{D \lambda}{D \tilde{t}}=\tilde{K}(1-\lambda) \exp (-\tilde{E} /(R \tilde{T}))
$$

where $\tilde{E}$ is the activation energy, $R$ is the universal gas constant, $\tilde{T}$ is the temperature, $\tilde{K}$ is a constant which is determined by assigning $l_{1 / 2}$, and $\lambda$ is the reaction progress variable defined as in Short and Stewart. ${ }^{5}$ The tildes represent dimensional values. The heat release of the decomposition reaction is $\tilde{Q}$, positive when exothermic. The gas is both thermally and calorically perfect, so that the internal energy is

$$
\tilde{e}=\frac{\tilde{p}}{(\gamma-1) \tilde{\rho}}-\tilde{Q} \lambda .
$$

The set of non-dimensionalizing scales is composed by the post-shock density, pressure, speed of sound and temperature, the already defined half reaction length and the ratio between length and velocity scale for the time scale. The non-dimensional detonation structure is thus specified by assigning the ratio of specific heats, and the three bifurcation parameters, which include the detonation overdrive, $f=M_{0}^{2} / M_{c j}^{2}$, the reduced activation energy, $E=\tilde{E} \gamma /{\tilde{c_{0}}}^{2}$, and the non-dimensional heat release, $Q=\tilde{Q} \gamma / \tilde{c_{0}}{ }^{2}$. Here $\tilde{c_{0}}$ denotes the pre-shock speed of sound, $M_{0}$ is the (pre-shock) detonation Mach number and $M_{c j}$ is the detonation Mach number that supports sonic conditions in the burnt stream. For the problem of detonation-turbulence interaction, an additional non-dimensional parameter is considered. This relates the longitudinal scale of turbulence to the reaction half length and is identified by the variable $L^{*}$ : the ratio between turbulence longitudinal length and reaction half distance. The limiting solution for $L^{*} \rightarrow \infty$ leads to Jackson, et al.'s ${ }^{2}$ turbulence-detonation analysis. The limiting solution $Q \rightarrow 0$ corresponds to Ribner's ${ }^{1}$ turbulence-shock interaction. Both Jackson, et al.'s and Ribner's solutions are independent of the detonation structure and thus of the activation energy.

\section{Pre-Shock Turbulence}

The pre-shock turbulence field is assumed to be isotropic, frozen and divergence-free so that the velocity field is obtained as the superimposition of planar vorticity (shear) waves as described in more detail by Ribner. ${ }^{1}$ The velocity perturbation of a shear component is parallel to the wave number vector $\vec{k}$. Due to cylindrical symmetry considerations, a shear component is identified (only) by its longitudinal velocity component $u_{0}$ (projection on the $x_{1}$ axis), the magnitude of the wave number vector $k$ and the angle between the wave vector and the shock plane $\theta_{0}$. Following Ribner, the von Kármán spectral model is used to determine the ratio of the longitudinal velocity spectral density to its mean square value. The von Kármán model $^{6}$ is best suited for high Reynolds number turbulence. In spherical coordinates $\left(k, \theta_{0}, \phi\right)$,

$$
\frac{[u u]_{0}}{\bar{u}^{2}{ }_{0}}=\frac{\hat{B} \hat{k}^{2} \cos ^{2} \theta_{0}}{2 \pi\left(1+\hat{k}^{2}\right)^{17 / 6}}
$$

where,

$$
\hat{k}=k \hat{a} L^{*}, \hat{B}=\frac{55}{18 \pi \hat{a}}, \hat{a}=\frac{55}{27 \pi} \mathscr{B}(1 / 3,5 / 2),
$$

and $\mathscr{B}$ denotes the Beta function.

\section{Post-Shock Turbulence}

The amplification of a wave pattern as it goes through the detonation is estimated in terms of autocorrelations. We seek to determine post-shock turbulence auto-correlations by integrating the "transferred" 
wave amplitudes over the pre-shock wave number space. The evaluation of the transfer functions will be shown in detail in $\S \mathrm{V}$. The post-shock turbulence is homogeneous on planes parallel to the unperturbed shock front (orthogonal to $x_{1}$ ) and in time. A scalar field $\alpha(\vec{x}, t)$ is expanded over the $\left(x_{2}, x_{3}, t\right)$ space in Fourier-Stieltjes series in the general form

$$
\alpha(\vec{x}, t)=\int e^{i\left[k_{2}, k_{3}, k_{t}\right]^{T}\left[x_{2}, x_{3}, t\right]} \mathrm{d} Z_{\alpha}\left(k_{2}, k_{3}, k_{t}, x_{1}\right),
$$

where $k_{2}$ and $k_{3}$ are the wave number vector projections onto the respective Cartesian axes, and $k_{t}=k_{1} D_{s}$, where $D_{s}$ is the detonation Mach number based on the post-shock speed of sound. Considerations about the homogeneity of the scalar field (see, for example, Moyal ${ }^{7}$ ) lead to expressing the auto-correlation as an integral over the wave number space,

$$
\overline{\alpha^{2}}\left(x_{1}\right)=\iiint[\alpha \alpha]\left(k_{2}, k_{3}, k_{t}, x_{1}\right) \mathrm{d} \hat{k}_{2} \mathrm{~d} \hat{k}_{3} \mathrm{~d} \hat{k}_{t} ;
$$

where $[\alpha \alpha]$ is the spectral density over $\left(\hat{k}_{2}, \hat{k}_{3}, \hat{k}_{t}\right)$. The transfer function $X_{\alpha}$ is defined such that

$$
[\alpha \alpha] \mathrm{d} \hat{k}_{2} \mathrm{~d} \hat{k}_{3} \mathrm{~d} \hat{k}_{t}=\left|X_{\alpha}\right|^{2}[u u]_{0} \mathrm{~d} \hat{k}_{1} \mathrm{~d} \hat{k}_{2} \mathrm{~d} \hat{k}_{3}
$$

so that integration is performed over the pre-shock wave number field. Integration over $\hat{k}_{2}$ and $\hat{k}_{3}$ defines the one-dimensional power spectrum,

$$
\Phi_{\alpha}=\iint\left|X_{\alpha}\right|^{2} \frac{[u u]_{0}}{\bar{u}_{0}^{2}} \mathrm{~d} \hat{k}_{2} \mathrm{~d} \hat{k}_{3}
$$

Substitution of equation (1) in equation (5) and conversion from Cartesian to spherical coordinates ${ }^{1}$ lead to

$$
\Phi_{\alpha}=\frac{\tilde{B}}{\left|\hat{k}_{1}\right|^{5 / 3}} \int\left|X_{\alpha}\right|^{2} \frac{\cos ^{3} \theta_{0}}{\sin ^{5} \theta_{0}\left(\hat{k}_{1}^{-2}+\sin ^{-2} \theta_{0}\right)^{17 / 6}} \mathrm{~d} \theta_{0} .
$$

Note that the power spectra are integrated in $\hat{k}_{1} \in[-\infty, \infty]$.

The transfer function depends on the wave number $\vec{k}$. This is different from the two limiting cases of Jackson, et al. ${ }^{2}$ and Ribner ${ }^{1}$ where it was a function of the angle $\theta_{0}$ only. The dependence of the transfer function on $k$ highlights the importance of the turbulence scaling effects, here represented by the variable $L^{*}$, which were ignored in previous discussions. It will be shown that the eigenvalues of the linear interaction homogeneous problem play a significant role on the dependency of the transfer functions on the wave number.

\section{Linearized Perturbation Equations}

The linear perturbation equations in the post-shock region are obtained from the compressible Euler reactive equations by ignoring second-order contributions and setting the time derivative operator,

$$
\frac{\partial}{\partial t}=V \frac{\partial}{\partial y}
$$

where $V=u_{0} \tan \theta_{0}$ is the velocity of the steady inertial reference frame defined by Ribner. ${ }^{8}$ The perturbation equations are written with respect to a reference system lying on a plane perpendicular to the shock plane and containing the wave number vector $\vec{k}$. In this system, $x$ (without subscript) is the direction normal to the shock and $y$ is the direction parallel to it. The component of the wave number vector on the $y$ axis is $k_{y}=k\left|\cos \left(\theta_{0}\right)\right|$. The post-shock turbulence components are thus determined in the cylindrical reference system $(x, y, \phi)$ with associated wave number components $\left(k_{1}, k_{y}, 0\right)$. The transverse wave number $k_{y}$ is 
unchanged across the shock, while the longitudinal is different from the pre-shock analog. In the steady reference frame attached to the shock wave, the post-shock mean solution features the two flow angles,

$$
\theta_{1} \equiv \tan ^{-1}\left[\tan \theta_{0} \frac{M_{1}^{2}(\gamma-1)+2}{(\gamma+1) M_{1}^{2}}\right]
$$

immediately after the shock, and

$$
\theta_{\infty} \equiv \tan ^{-1}\left[\frac{\tan \theta_{0}\left(2+(\gamma-1) M_{1}^{2}\right)}{1+\gamma M_{1}^{2}-\sqrt{\left(1-M_{1}^{2}\right)^{2}-2 Q c_{0}^{2} / \gamma\left(\gamma^{2}-1\right) M_{1}^{2}}}\right],
$$

in the far-field. Here, $M_{1}$ is the Mach number immediately after the shock, which is a function of the pre-shock Mach number $M_{0}$ only, or, by means of a more complex expression, of the detonation structure parameters,

$$
M_{1}^{2}=\frac{f(\gamma-1)\left(\gamma Q-Q / \gamma+\sqrt{\left(\gamma^{2}-1\right) Q\left(2 \gamma+\left(\gamma^{2}-1\right) Q\right)} / \gamma+1\right)+2}{-\gamma+2 f\left(\gamma\left(\gamma Q+\sqrt{\left(\gamma^{2}-1\right) Q\left(2 \gamma+\left(\gamma^{2}-1\right) Q\right)} / \gamma+1\right)-Q\right)+1}
$$

The solution array is composed by the complex transfer functions,

$$
z \equiv\left[X_{\rho}, X_{u}, X_{v}, X_{p}, X_{\lambda}\right]^{T},
$$

plus the ratio between the shock corrugation angle and the longitudinal velocity component of the pre-shock shear wave $\sigma$. Denoting by $Z$ the mean-flow solutions,

$$
Z \equiv[\rho, u, v, p, \lambda]^{T}
$$

we obtain the following vector equation,

$$
\boldsymbol{A} \frac{\partial z}{\partial x}+(\boldsymbol{B}+V \boldsymbol{D}) \frac{\partial z}{\partial y}+\boldsymbol{C} z-(\boldsymbol{B}+V \boldsymbol{D}) \frac{\partial Z}{\partial x} \sigma=0
$$

The $5 \times 5$ matrices denoted with bold typeface are

$$
\begin{gathered}
\boldsymbol{A} \equiv\left[\begin{array}{ccccc}
u & \rho & 0 & 0 & 0 \\
0 & u & 0 & \frac{1}{\gamma \rho} & 0 \\
0 & 0 & u & 0 & 0 \\
0 & T-u^{2} & 0 & 0 & 0 \\
0 & 0 & 0 & 0 & u
\end{array}\right], \quad \boldsymbol{B} \equiv\left[\begin{array}{ccccc}
0 & 0 & \rho & 0 & 0 \\
0 & 0 & 0 & 0 & 0 \\
0 & 0 & 0 & \frac{1}{\gamma \rho} & 0 \\
0 & 0 & T & 0 & 0 \\
0 & 0 & 0 & 0 & 0
\end{array}\right], \\
\boldsymbol{C} \equiv\left[\begin{array}{ccccc}
\frac{\partial u}{\partial x} & \frac{\partial \rho}{\partial x} & 0 & 0 & 0 \\
-\frac{\partial p}{\partial x} /\left(\rho^{2} \gamma\right) & u & 0 & 0 & 0 \\
0 & 0 & 0 & 0 & 0 \\
\zeta r_{\rho}-\frac{T}{\rho} \frac{\partial u}{\partial x} & -2 u \frac{\partial u}{\partial x} & 0 & \zeta r_{p}+\frac{\partial u}{\partial x} / \rho & \zeta r_{\lambda} \\
-r_{\rho} & \frac{\partial \lambda}{\partial x} & 0 & -r_{p} & -r_{\lambda}
\end{array}\right], \quad \boldsymbol{D} \equiv\left[\begin{array}{ccccc}
1 & 0 & 0 & 0 & 0 \\
0 & 1 & 0 & 0 & 0 \\
0 & 0 & 1 & 0 & 0 \\
0 & -u & 0 & \frac{1}{\rho \gamma} & 0 \\
0 & 0 & 0 & 0 & 1
\end{array}\right] .
\end{gathered}
$$

where $\zeta=-Q c_{0}^{2}(\gamma-1) / \gamma$ and the derivatives of the rate term with respect to density, pressure, and progress variable are:

$$
r_{\rho}=-r E \frac{c_{0}^{2}}{p}, \quad r_{p}=r E \frac{\rho c_{0}^{2}}{p^{2}}, \quad r_{\lambda}=-K \exp \left(-E \frac{\rho c_{0}^{2}}{p}\right) .
$$

The evaluation of the auto-correlation in the wave number space, equation (3), and the linearity of the problem allow us to analyze the Fourier components individually, so that the substitution $\partial z / \partial y \rightarrow i k_{y} z$ yields a system of ordinary differential equations in $x$. The linear system requires six boundary conditions, 
five are imposed at the shock front, and one at the far-field, $x \rightarrow \infty$. The shock boundary conditions are obtained by linearization of the Rankine-Hugoniot shock relations. They are written in vector form as

$$
z(\gamma+1)=\boldsymbol{S}_{a} \frac{\cos \theta_{1} / \cos \theta_{0}}{\sqrt{4 \cos ^{2} \theta_{1}\left(1-M_{1}^{2}\right)\left(\gamma M_{1}^{2}+1\right)+M_{1}^{4}(\gamma+1)^{2}}}+\boldsymbol{S}_{\boldsymbol{b}} \frac{\tan \theta_{1}}{M_{1}^{2}(\gamma-1)+2} \sigma,
$$

where the $5 \times 1$ arrays on the right-hand side are defined below,

$$
\begin{gathered}
\boldsymbol{S}_{\boldsymbol{a}} \equiv\left[\begin{array}{c}
4 / M_{1}(1-\gamma)+8 \gamma M_{1} \\
\left(M_{1}^{2}\left(\gamma^{2}-6 \gamma+1\right)+4(\gamma-1)\right) \sec \theta_{1} \\
4\left(1+\gamma\left(2 M_{1}^{2}-1\right)\right) \tan \theta_{1} \\
4 \gamma M_{1}\left(M_{1}^{2}(\gamma-1)+2\right) \\
0
\end{array}\right], \\
\boldsymbol{S}_{\boldsymbol{b}} \equiv\left[\begin{array}{c}
-4\left(1+\gamma\left(2 M_{1}^{2}-1\right)\right) \\
4 / M_{1}\left((1-2 \gamma) M_{1}^{4}+(\gamma-3) M_{1}^{2}+\left(\gamma M_{1}^{4}+1\right) \cos \left(2 \theta_{1}\right)+1\right) \csc \left(2 \theta_{1}\right) \\
-4 \gamma M_{1}^{2}\left(M_{1}^{2}(\gamma-1)+2\right) \\
0
\end{array}\right] .
\end{gathered}
$$

The far-field condition is obtained by imposing no left-running characteristics, practically nullifying the upstream traveling acoustic wave. This condition is similar to that proposed by Short and Stewart, ${ }^{5}$ but here we include the dependence of the solution on $\lambda$. In order to do so, we replace in equation (8)

$$
\frac{\partial z}{\partial x} \rightarrow \alpha z, \quad \frac{\partial z}{\partial y} \rightarrow i k_{y} z
$$

and solve for the $\alpha$ (generalized) eigenvalues and the associated right eigenvectors matrix, $R$. The final condition is obtained by substituting in the eigenvectors matrix the column corresponding to the upstream acoustic with the solution vector $-z$ and imposing that the determinant of such matrix is equal to zero. In a vector form, we write $\boldsymbol{S}_{\boldsymbol{c}}{ }^{T} z=0$, where

$$
\begin{gathered}
\boldsymbol{S}_{\boldsymbol{c}} \equiv\left[\begin{array}{c}
0 \\
S_{d 2} S_{e 5} \\
S_{d 3} S_{e 5} \\
S_{d 4} S_{e 5} \\
\boldsymbol{S}_{\boldsymbol{d}}^{T} \boldsymbol{S}_{\boldsymbol{e}}
\end{array}\right], \quad \boldsymbol{S}_{\boldsymbol{d}} \equiv\left[\begin{array}{c}
0 \\
V \\
-u \\
i /(\rho \gamma) \sqrt{1-M_{w}^{2}} \\
0
\end{array}\right], \\
\boldsymbol{S}_{\boldsymbol{e}} \equiv\left[\begin{array}{c}
0 \\
r_{\lambda} u\left(-i r_{\lambda}-k_{y} V\right) \zeta \\
k_{y} r_{\lambda} u^{2} \zeta \\
i \zeta r_{\lambda}^{2} u^{2} \rho \gamma \\
i T\left(\left(u^{2}+V^{2}\right) k_{y}^{2}+2 i r_{\lambda} V k_{y}+r_{\lambda}^{2}\left(M_{\infty}^{2}-1\right)\right)
\end{array}\right],
\end{gathered}
$$

and $M_{w} \equiv \sqrt{\left(u_{\infty}^{2}+V^{2}\right) / T_{\infty}}$ and $M_{\infty} \equiv \sqrt{u_{\infty}^{2} / T_{\infty}}=M_{w} \cos \theta_{\infty}$ are the far-field (burnt gas side) Mach numbers in the steady and shock reference frames, respectively. Note: all the flow variables in equation (14) are evaluated in the far-field and the superscript $\infty$ is dropped for brevity of notation.

\section{V.A. Far-Field Conditions}

Far-field conditions are associated with the characteristic waves in the constant property $(\lambda=1)$ region. The contributions to the perturbation field $(z)$ of these waves will be studied in detail and denoted as acoustic 
and entropic contributions. The characteristic polynomial associated with a two-dimensional system in equation (8) has a double root in the constant property far-field. It is customary to associate to the double root a zero velocity entropy wave and a constant property vorticity wave. In the present context, entropy and vorticity waves arise as result of the shock-turbulence interaction; therefore, they are not independent of each other. For this reason, the entropy wave is defined here as the sum of the wave contributions associated with the double root. Since in the far-field derivatives of the mean flow variables are zero, the entries in the first column, rows 2-5 of matrices $\boldsymbol{A}, \boldsymbol{B}, \boldsymbol{C}$ and $\boldsymbol{D}$ are all zeros. This condition leads to a decoupled eigenvalue problem (in $\alpha$ ) obtained by eliminating the first row and first column from equation (8). The outcome of the system reduction is that the velocity and pressure contributions from the acoustic and entropy waves are identified by the eigenvectors of the decoupled problem. An additional contribution to the density associated with the entropy wave is determined by subtracting the local density to the density term evaluated using the first of equation (8) and the acoustic velocity terms. Thus, the post-shock field is decomposed into an isentropic acoustic field and an entropy varying solenoidal field (variable density shear pattern), while the pre-shock perturbation field is assumed to be solenoidal and isentropic (constant density shear pattern). The acoustic field is responsible for the noise while the entropy field is responsible for the spottiness behind the wave.

Let the operator $\operatorname{Diag}^{\operatorname{Re}=0}[w]$ applied to a generic vector $w$ form a diagonal matrix having on the main diagonal the vector entries $w_{i}$ with number corresponding to the eigenvalues $\alpha_{i}$ with zero real part, and zeros elsewhere. The far-field contributions associated with the $\alpha$ eigenvalues are the columns of the matrix

$$
\boldsymbol{F} \equiv \boldsymbol{R} \operatorname{Diag}^{\operatorname{Re}=0}\left[\boldsymbol{R}^{-1} z_{\infty}\right]
$$

The array $z_{\infty}$ is evaluated at the downstream boundary of the solution domain, the extension of which is detailed in $\S$ VII. The entropy wave eigenvalue, $\alpha_{1} \equiv-i k_{y} \tan \theta_{\infty}$, is a purely imaginary number for all values of $\theta_{0}$, thus entropy contributions created in the induction region do not decay in the far-field. This statement can be easily viewed as a consequence of Crocco's equation for an inviscid steady field, as per Ribner. ${ }^{8}$ The downstream running acoustic eigenvalue,

$$
\alpha_{2} \equiv k_{y} \frac{i M_{\infty}^{2} \tan \theta_{\infty}-\sqrt{1-M_{\infty}^{2} \sec ^{2} \theta_{\infty}}}{1-M_{\infty}^{2}}
$$

is imaginary only for values of upstream angle

$$
\theta_{0}>\tan ^{-1}\left(\frac{u_{\infty}}{u_{0}} \sqrt{M_{\infty}^{-2}-1}\right) \equiv \theta_{c r} .
$$

The critical value can be shown to be dependent on $\gamma, Q$ and $f$, and is identical to the value found in Jackson, et al. ${ }^{2}$ Acoustic contributions decay in the far-field for $\theta_{0}<\theta_{c r}$. It is easily verified that for overdrive values approaching unity, the free-stream Mach number approaches unity and $\theta_{c r} \longrightarrow 0$.

\section{Homogeneous Problem}

The homogeneous problem associated with the turbulence amplification problem described in $\S \mathrm{V}$ is obtained by setting $\boldsymbol{S}_{\boldsymbol{a}}=0$ in equation (12). The homogeneous problem is solved for complex eigenvalues $k_{y}$. Of particular importance are values of $k_{y}{ }^{*}$ with zero imaginary parts. The set of $k_{y}{ }^{*}$, and their longitudinal counterparts $k_{1}{ }^{*}$, form a set of natural, characteristic wave numbers associated with a detonation structure. In the turbulence amplification problem, where non-homogeneous boundary conditions are considered and the wave numbers are real, the transfer functions grow unbounded for values of $k_{y}$ approaching $k_{y}{ }^{*}$. Thus $k_{y}{ }^{*}$ are singular values in the one-dimensional power spectra. For a given detonation structure $(f, Q, E$ and $\gamma)$, $k_{y}{ }^{*}$ eigenvalues are sought by varying the angle $\theta_{0}$ in $[0, \pi / 2]$.

The conditions for the existence of real eigenvalues can be related to the two-dimensional stability problem of a detonation wave. If one of such eigenvalues exists, it is easily proved that the two-dimensional instability problem of Short and Stewart ${ }^{5}$ has a neutral point (a zero growth rate solution) with phase speed $V$. That this is the case can be shown by direct comparison of the equations reported by Short and Stewart ${ }^{5}$ with 
those presented in the previous section. More intuitively, neutral solutions are sinusoidal waves traveling along the $y$-axis at the phase speed, so that an observer moving with speed $V$ in the negative $y$ direction would experience a steady perturbation field. The analogy between the two-dimensional instability problem and the homogeneous problem proves that only two-dimensionally unstable detonations admit $k_{y}{ }^{*}$ values, and that such eigenvalues identify freely oscillating (natural) solutions.

Since the effect of the structure on the post-detonation turbulence amplification is maximum at $k_{y}{ }^{*}$, we are mainly interested in detonation conditions close to the stability limit. For weakly stable detonations, we will focus on the power spectra amplification at wave numbers equal to the real part of eigenvalues with minimum imaginary part. The parallel between the homogeneous problem and the two-dimensional stability problem is completed by noticing that conditions for which $k_{y}$ eigenvalues with negative imaginary parts exist identify detonation structures that are two-dimensionally unstable.

\section{Numerical Method}

Equation (8) is discretized in the $x$ direction using the Chebyshev tau method. ${ }^{9}$ The generalized eigenvalue problems discussed in $\S \mathrm{VI}$ are solved using a QZ algorithm ${ }^{10}$ by means of which all the spatial eigenvalues and eigenvectors are determined. Denoting by $N$ the order of the maximum Chebyshev polynomial resolved in the truncation error, and $C_{k}(x)$ the Chebyshev polynomial of the $k_{t h}$ order, the expansion takes the form:

$$
z_{j}=\sum_{k=0}^{N+1} \hat{z}_{j, k} C_{k}\left(-1+2 \frac{x}{L_{d}}\right)
$$

where $\hat{z}_{j, k}$ is the array of Chebyshev components and $L_{d}$ is the total length of the solution domain in the post-shock region, determined as $L_{d}=1.25 \times x_{\infty}$. Here $x_{\infty}$ is the $x$ location that corresponds to a progress variable value, $\lambda=1-1 \times 10^{-8}$. The mean flow solution is assumed to be constant for $x>x_{\infty}$.

\section{Results}

\section{VIII.A. Verification}

The numerical approach is verified against Ribner's ${ }^{1}$ solution for a $M_{0}=1.25, \gamma=1.2$ non-reacting shock. Ribner's formulae given in Appendix $\mathrm{A}^{1}$ were implemented with almost no change but for the value of $\beta^{2}$ which is taken to be $1-M_{1}^{2}$ rather than $1-M_{0}^{2}$.

Chebyshev expansion solutions are obtained using $Q=1 \times 10^{-9}, E=10$, and $f=M_{0}^{2}$. The onedimensional spectrum is evaluated by integrating equation (6) over $\theta_{0} \in[0, \pi / 2]$ using an adaptive Simpson quadrature with a tolerance of $1 \times 10^{-6}$. Results of this validation are shown in Fig. 1, which refers to the absolute error in the longitudinal velocity component of the post-shock turbulence. Panels $(\mathrm{a}, \mathrm{b})$ and $(\mathrm{d})$ show that, in the free-stream and at the shock front, the analytical solution is obtained to integration tolerance for all values of the expansion order. This result is expected based on the fact that Ribner's solution ${ }^{1}$ can be simply obtained by imposing no left running acoustic at the shock, i.e., the non-reactive problem can be solved using only equations (12) and (14). This conclusion does not apply to the reactive flow case, for obvious reasons, and to the non-reactive solution at finite $x$ locations. Discretization error affects the Chebyshev expansion solution at finite locations. Panel (c) demonstrates that the absolute spectrum error is smaller than the integration tolerance for $N \approx 180$, which is selected as the expansion order for all numerical results presented in this section. Finally, we remark that on the scale of Fig. 1 even the velocity spectrum for $N=90$ is indistinguishable from the analytic analog.

\section{VIII.B. Activation energy effect on one-dimensional spectra}

We seek to identify the effect of the activation energy on the turbulence amplification and link it to changes in characteristic solutions of the associated homogeneous problem. Referring to the stability analysis, ${ }^{5}$ we start with a stable detonation structure close to the instability boundary by setting $Q=2, E=10, \gamma=1.2$, $f=1.2$, and $L^{*}=1$. Chebyshev expansion solutions are obtained for values of $E$ on either side of the stability 


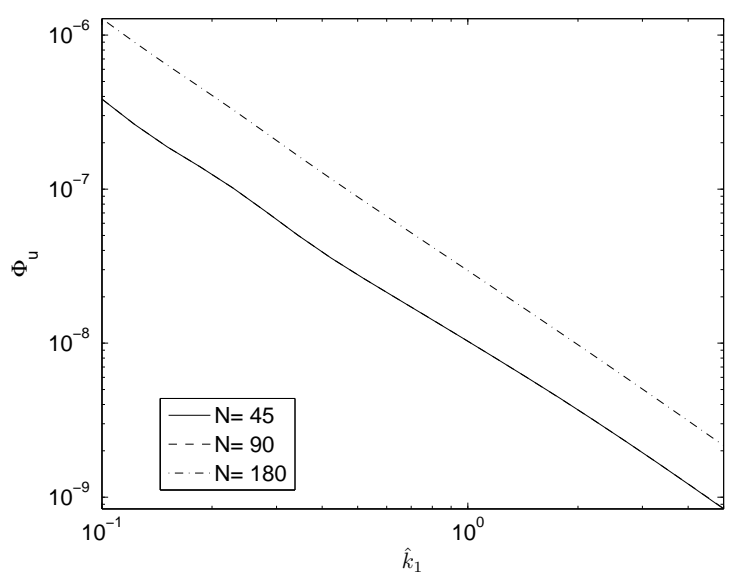

(a) Velocity Entropy Contribution

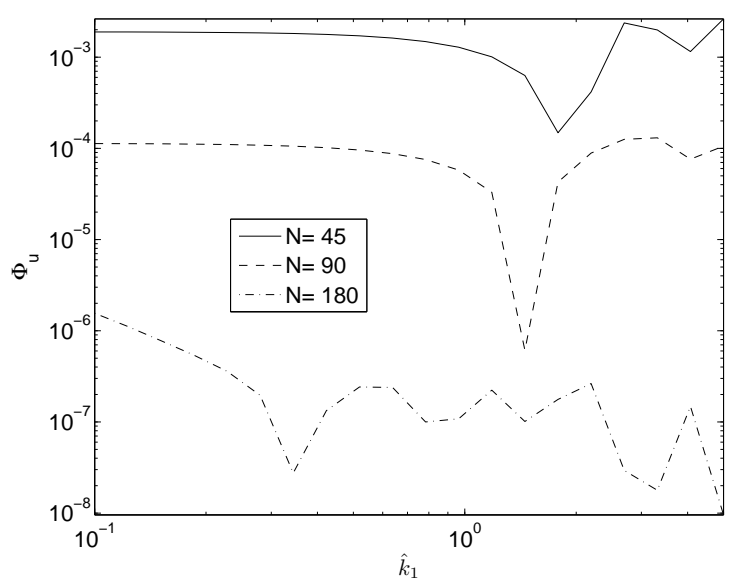

(c) Velocity at $x=1$

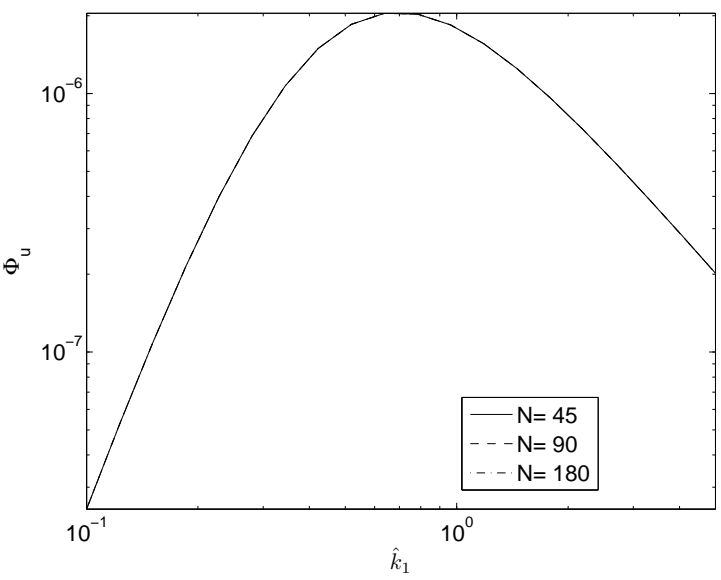

(b) Velocity Acoustic Contribution

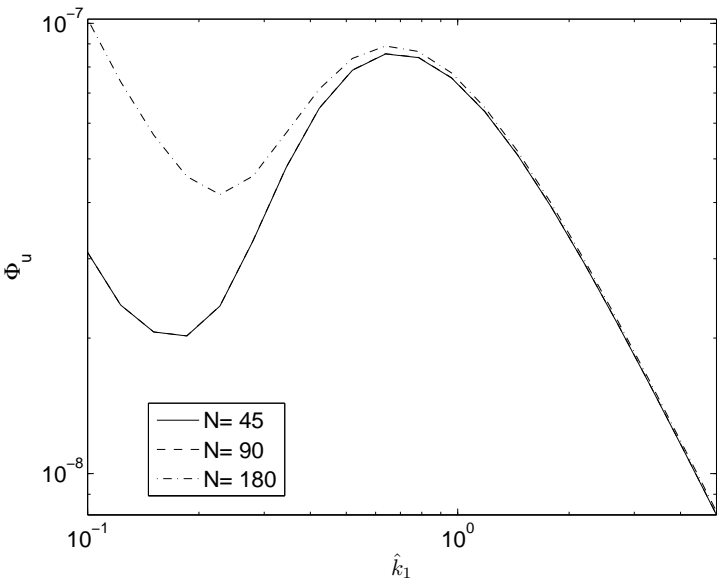

(d) Velocity at $x=0$

Figure 1. Absolute error in one-dimensional power spectrum between Chebyshev expansion and Ribner's ${ }^{1}$ analytical solutions. 
boundary with all the other parameters fixed. Solutions with $E=15$ (unstable), $E=5$ (stable) and $E=0$ (stable) are discussed. Results shown in Fig. 2 demonstrate the strong influence of the activation energy on the turbulence emanating from a reacting shock. The unstable $E=15$ solution manifests the presence of two $k_{y}{ }^{*}$ eigenvalues, both occurring at super-critical values of $\theta_{0}$; for any longitudinally stable (in the sense discussed in Short and Stewart ${ }^{5}$ ) eigenmode there are two $k_{y}{ }^{*}$ values corresponding to the neutral stability points. The super-critical nature of the eigenvalues implies that the acoustic far-field is strongly affected. Resonance augmentation of the longitudinal velocity spectra is substantial only for activation energies close to or higher than the instability threshold, $E \geq 10$; solutions for $E=5$ and $E=0$ are only marginally different both at the fire zone $(x=1)$ and in the far-field.

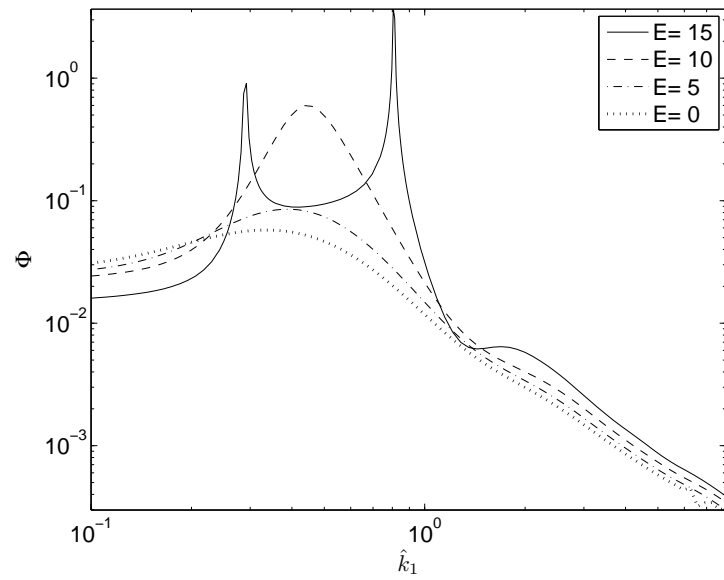

(a) Pressure at $x=1$

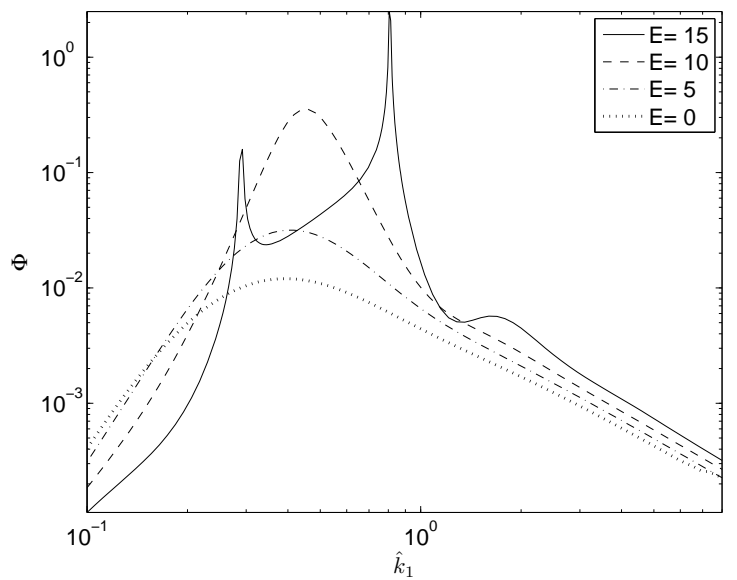

(c) Pressure Acoustic Contribution

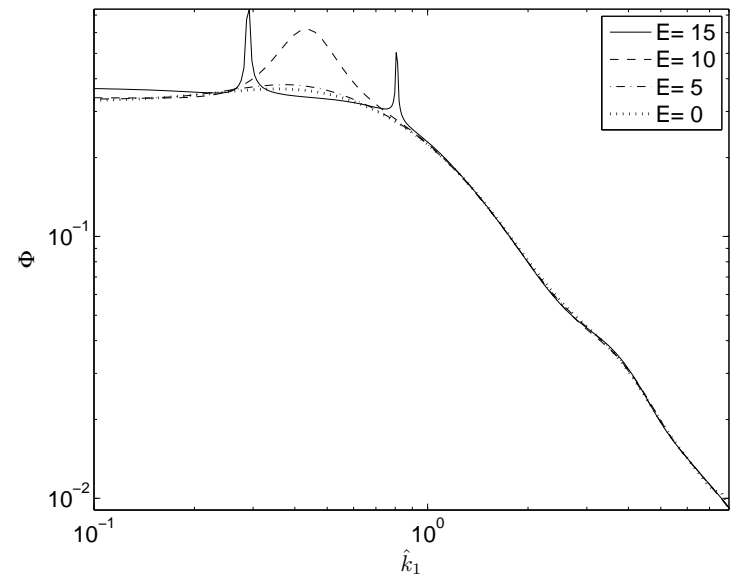

(b) Velocity at $x=1$

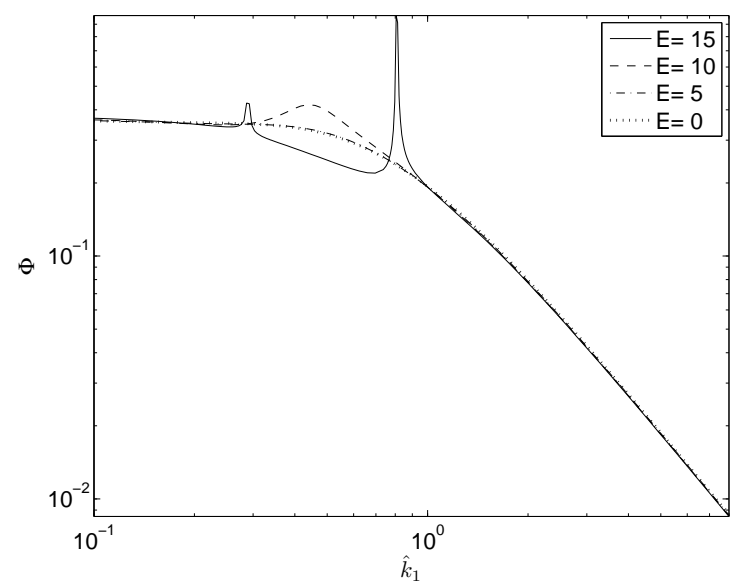

(d) Velocity Entropy Contribution

Figure 2. Variation of the power spectrum with the activation energy for a $Q=2, \gamma=1.2, f=1.2$, and $L^{*}=1$ structure.

The homogeneous problem eigenvalues with smallest imaginary part are plotted in Fig. 3 against $\theta_{0}$ for $E=10$ and $E=15$. The range of $\theta_{0}$ covers the interval $1.01 \theta_{c r}<\theta_{0}<1.5 \theta_{c r}$ so all shown eigensolutions are super-critical. The filled dots on the unstable $(E=15$, dashed line) curve denote the real eigenvalues, cf. Fig. 3(a). The associated purely real $\hat{k}_{1}$ values in Fig. $3(\mathrm{~b})$ match the locations of the power spectra 
cusps in Fig. 2. The stable detonation curve $(E=10)$ is marked in Fig. 3(a) in correspondence of the eigenvalue with minimum imaginary part ${ }^{\mathrm{a}}$. The associated real value in Fig. 3(b) (filled dot on the solid curve) corresponds with the value of maximum amplification of the power spectra in Fig. 2. Given that for the stable $E=10$ detonation the $\min \left(\Im\left\{k_{y}\right\}\right)$ eigenvalue is not a natural solution, the correspondence between maximum amplification and real eigenvalue is not exact: for the four spectra plotted in Fig. 2 the maximum location varies in the interval $\hat{k}_{1} \in[0.438,0.461]$, while the filled dot on the solid line in Fig. $3(\mathrm{~b})$ is at $\hat{k}_{1}=0.416$. Figure 3 establishes a strong relation between eigenvalues of the homogeneous system and power spectra post-shock amplification: longitudinal wave numbers close to the real part of eigenvalues with smallest imaginary component are maximally amplified; the activation energy plays a significant role in this phenomenon by moving poles close to the real axis.

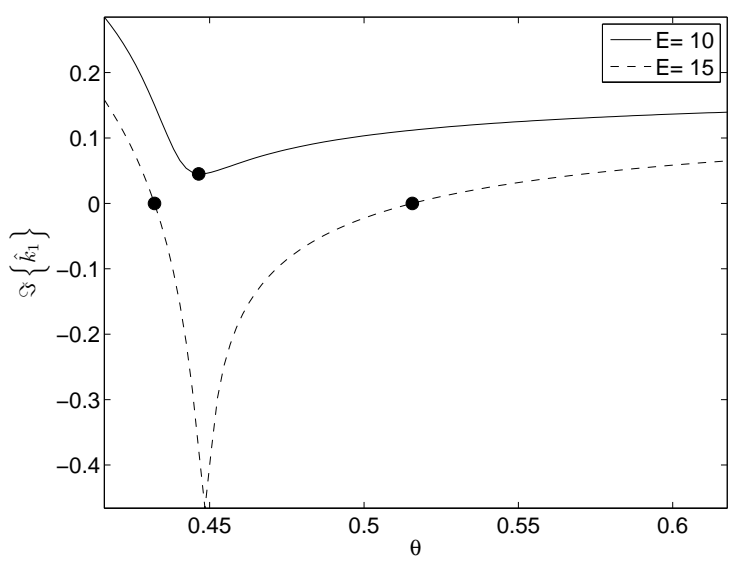

(a) Imaginary Part

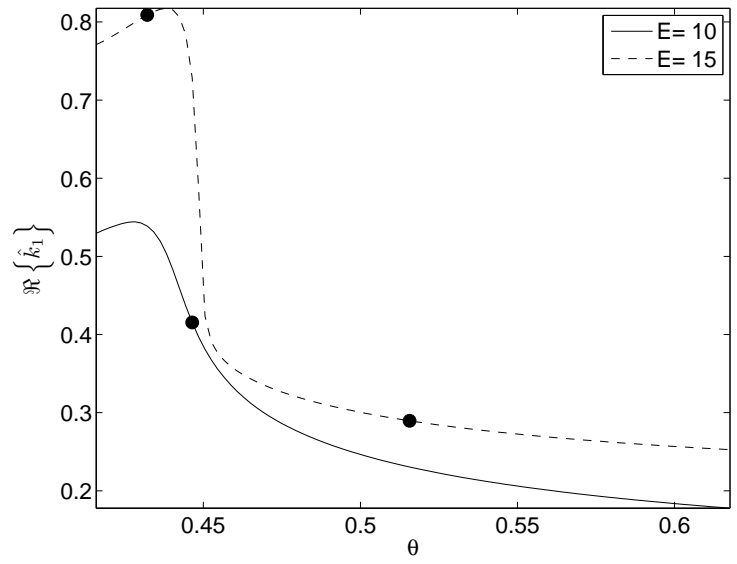

(b) Real Part

Figure 3. Homogeneous problem eigenvalues for two values of the activation energy.

The figures shown in this section suggest that much information about the post-shock turbulence can be gained by analyzing eigenvalues of the homogeneous problem. Figures 2 and 3 demonstrate that eigensolutions on the stability boundary well describe resonant phenomena in the turbulence-structure interaction for weakly stable detonations. The limiting conditions for which the $k_{y}$ eigenvalue with minimum imaginary part is real determine the stability boundary. The boundary identifies a surface in the $E, Q, f$ space. Figure 4 correlates $Q, \theta_{0} / \theta_{c r}, \hat{k}_{1}$ and $\theta_{\infty}$ with the activation energy on the stability boundary in the range $E \in[0,20]$ and for four overdrive values. Limiting values of the heat release for $f=1.2$ agree well with the values reported in the literature ${ }^{5}$ detonations with larger overdrives support a smaller limiting heat release for most activation energies investigated; the exception being values very close to 0 , Fig. 4(a). This conclusion challenges the notion that higher overdrives leads to stabilization of a detonation wave, and is somewhat in line with the work of Short and Stewart, ${ }^{5}$ who found that an increase in overdrive never leads to crossing the stability boundary.

The longitudinal wave number plotted in Fig. 4(c) determines conditions of maximum single wave amplification, a contribution that dominates the post-shock spectrum according to results shown in Fig. 2. Figure 4 (b) shows that such conditions occur in the vicinity of the critical point, $\theta_{0} \approx \theta_{c r}$. An increase in activation energy leads to an increased value of $\theta_{0} / \theta_{c r}$, while an increase in overdrive leads to a diminished value. Given that the critical angle increases with the overdrive on the stability boundary, refer to equation (16), the natural frequency angle $\theta_{0}$ actually increases with the overdrive. Results in Fig. 4(b) suggest that the acoustic field behind a detonation wave close to the stability boundary is significantly weaker for

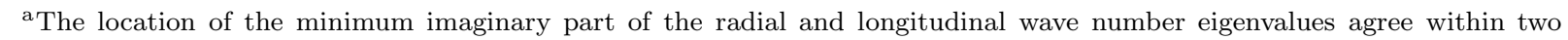
precision digits, thus the radial wave number is chosen to identify the minimum 
high values of the overdrive.

The value of the longitudinal pre-shock wave number $\hat{k}_{1} / L^{*}$ increases with the activation energy and decreases with the overdrive for sub-critical solutions. Thus structures with low activation energy and large overdrive are more likely to interact at a resonance level with large-scale turbulence, i.e., high values of $L^{*}$. The $\hat{k}_{1}$ curves are shown to be discontinuous against $E$ (in the first derivatives) when the the ratio $\theta_{0} / \theta_{c r}$ crosses unity, compare curves for $f=1.4$ and $f=1.6$ on Figs. 4(b) and 4(c). Figure 4(d) shows that the angle of the refracted entropy wave decreases with the increasing activation energy. Therefore, it behaves in an opposite way with respect to the incoming shear wave angle.

Temperature transfer eigenfunctions for four activation energy and four overdrive values are shown in Fig. 5. The eigenfunctions are obtained by setting the front pressure perturbation to 1 , and therefore represent ratios between temperature perturbations and pressure perturbations at the shock. The eigenfunctions are largest for low activation energy and overdrive values.

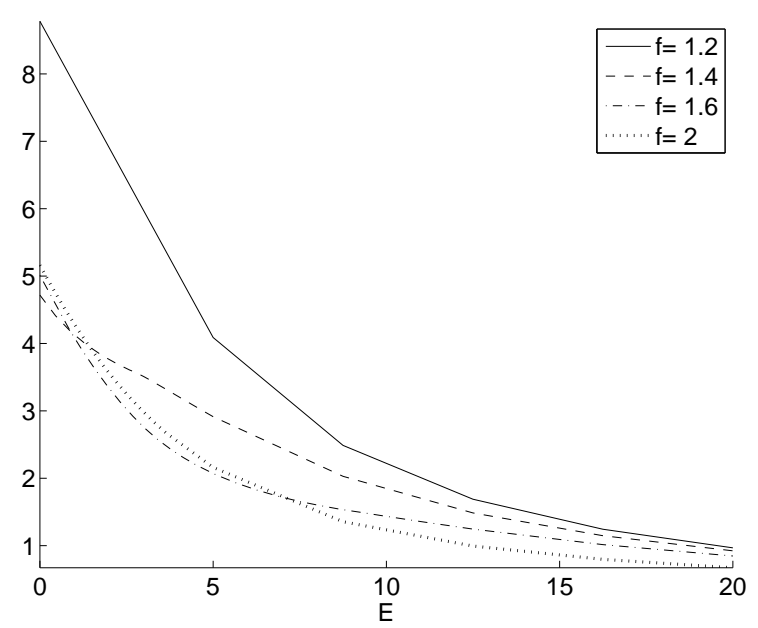

(a) Q

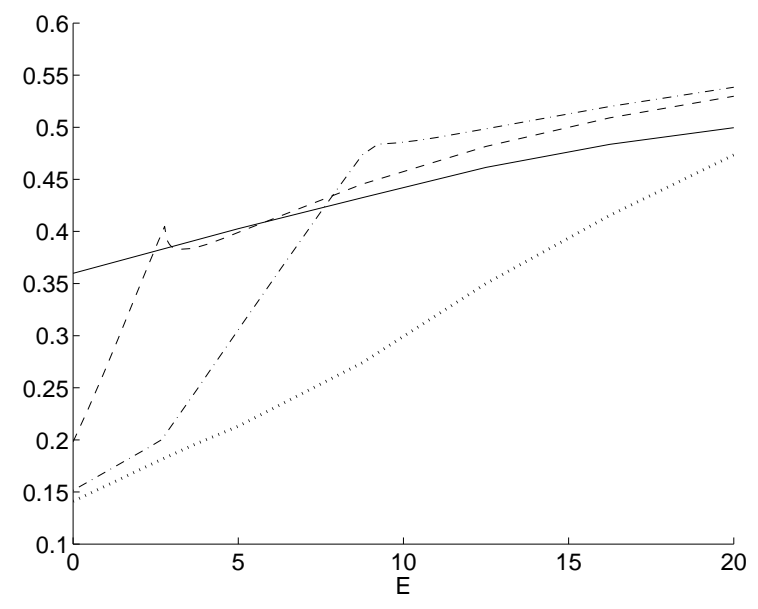

(c) $\hat{k}_{1} / L^{*}$

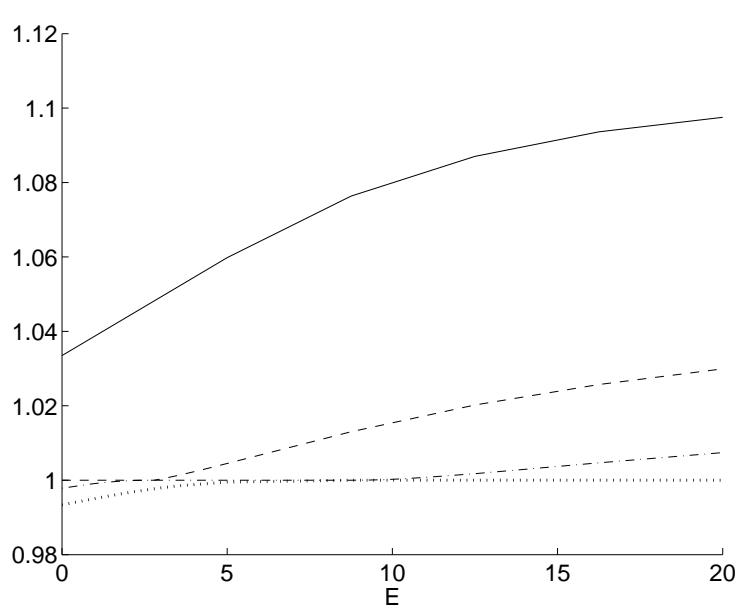

(b) $\theta_{0} / \theta_{c r}$

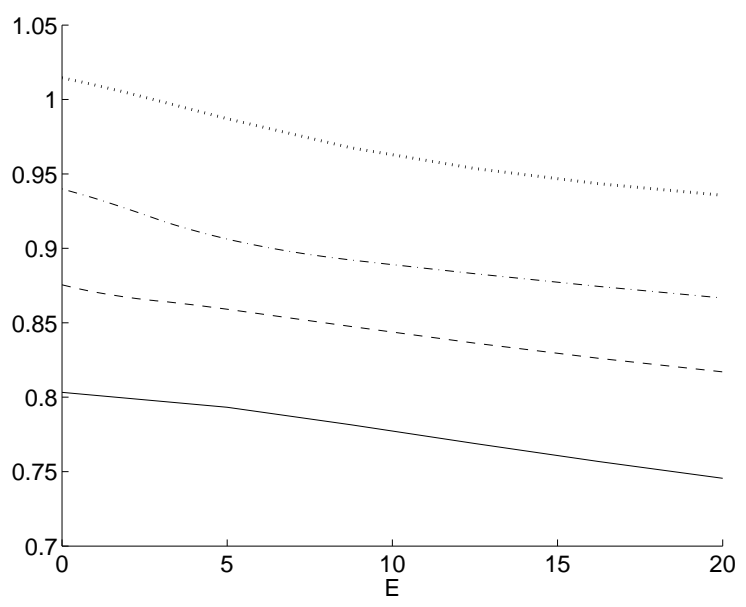

(d) $\theta_{\infty}$

Figure 4. Detonation conditions on the stability boundary. The four panels show $Q, \theta_{0} / \theta_{c r}$ and $\hat{k}_{1}$ and $\theta_{\infty}$ against the activation energy. The legend shown only in panel (a) applies to the four panels. 


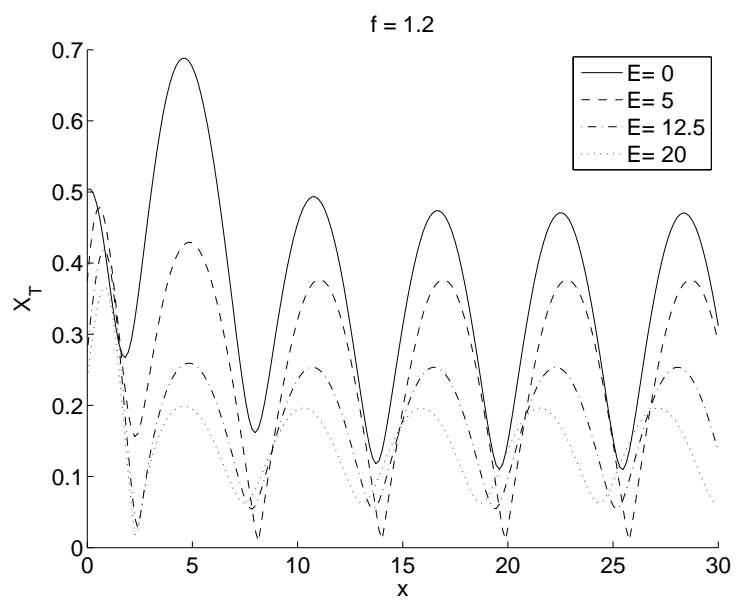

(a)

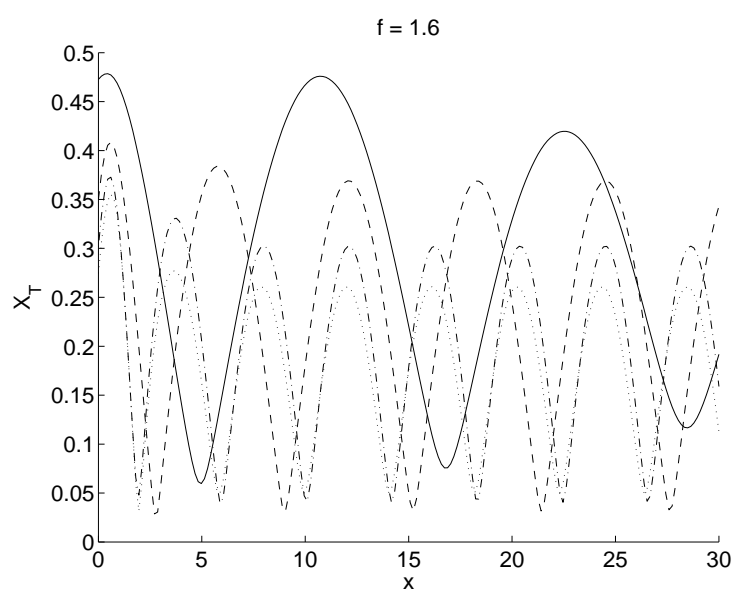

(c)

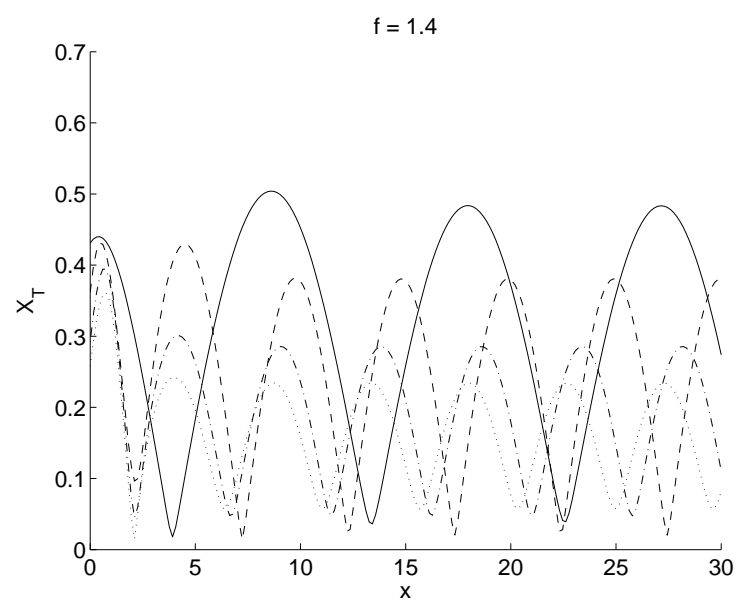

(b)

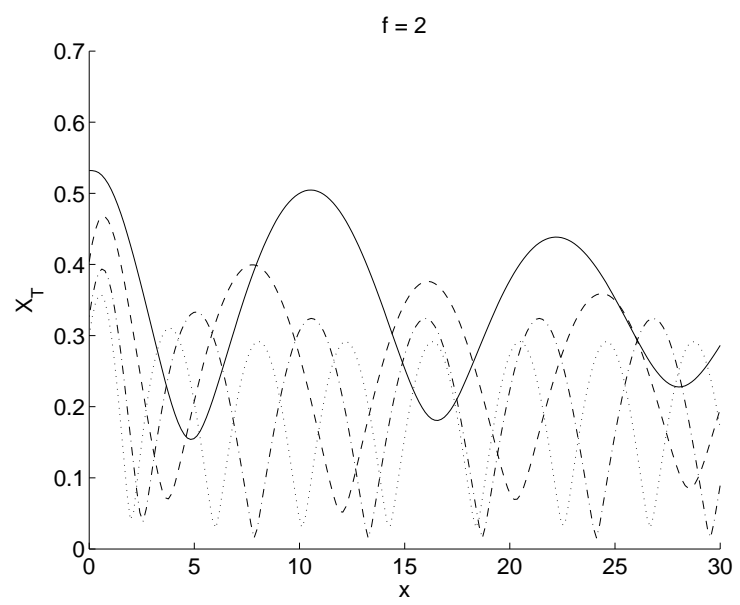

(d)

Figure 5. Temperature transfer eigenfunction, $X_{T}$, for four values of overdrive and activation energy. The legend shown only in panel (a) applies to the four panels.

Based on the characteristic solution analysis, a major effect of increasing the overdrive on the stability boundary is acoustic attenuation. For large overdrive and low activation energy the maximally amplified wave is sub-critical; a significant decrease in overdrive can cause such wave to become super-critical. Onedimensional spectra are obtained for two cases with $f=1.2$ and $2, E=2$ (in both cases) and $Q=0.999 Q_{*}$, where $Q_{*}$ denotes the value on the stability boundary. Results are displayed in Fig. 6 . The higher overdrive spectrum has substantially weaker post-shock acoustic fields and lacks strong amplification at the single $\hat{k}_{1}^{*}$ characteristic wave number. Both cases manifest a strong entropy field with a cusp-like feature close to the characteristic frequency.

\section{VIII.C. Structure Effect on Unstable Waves}

The extension of the analysis to unstable detonations requires considerations concerning the effect of the global instability on the power spectra. Under the assumptions of frozen turbulence and weak instability,

\section{2 of 20}




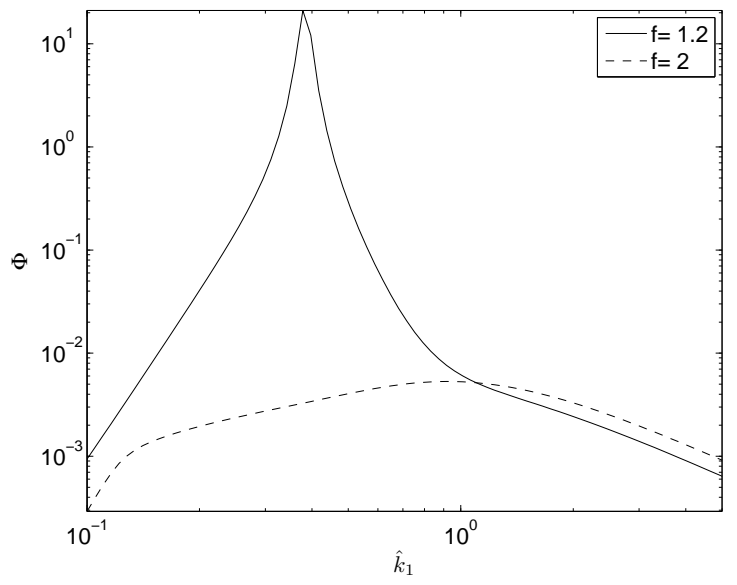

(a) Pressure Acoustic Contribution

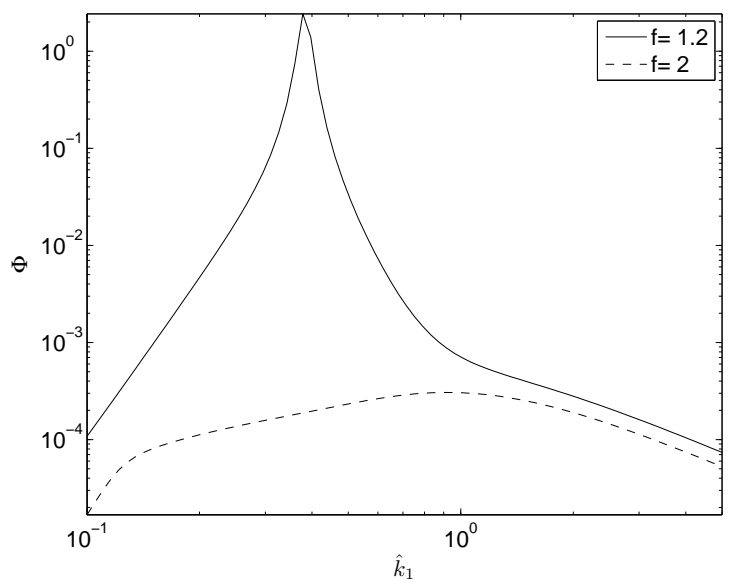

(c) Temperature Acoustic Contribution

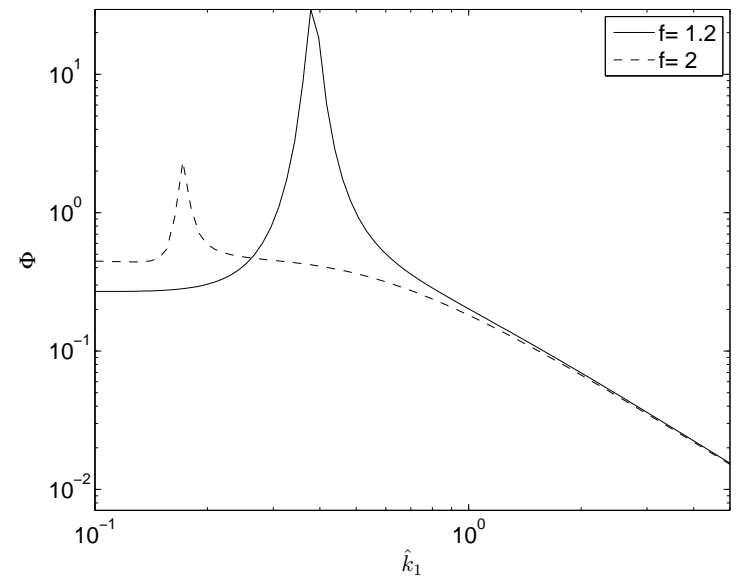

(b) Velocity Entropy Contribution

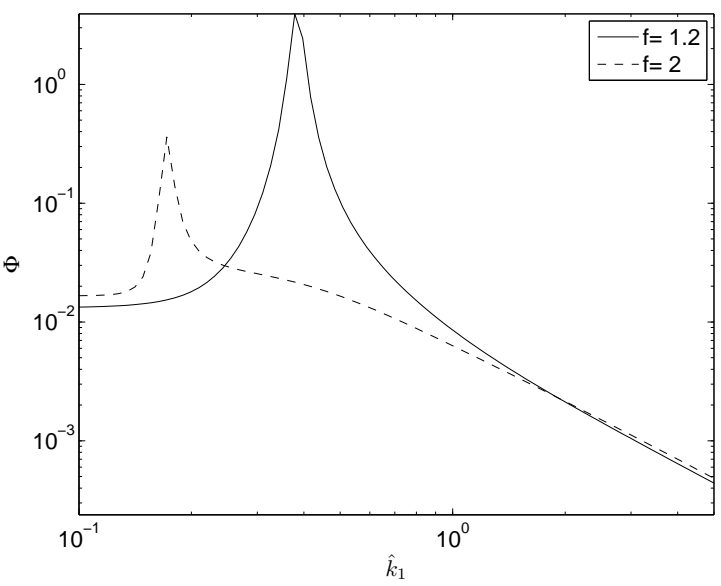

(d) Temperature Entropy Contribution

Figure 6. One-dimensional power spectra for two detonation structures near the stability boundary. Solid curve: $E=2$, $f=1.2, Q=0.999 \times 6.4674$. Dashed curve: $E=2, f=2, Q=0.999 \times 3.5597$. 
the large time post-shock perturbation field is affected by the global instability in two ways: first, the mean flow is time-dependent, second, the convected noise is superimposed to a non-forced, intrinsic field, result of limit-cycle oscillations, and thus independent of the pre-shock field. The convected noise will also be denoted as forced response, because it corresponds to the particular solution of the inhomogeneous (boundary-forced) system in equation (8) devoid of intrinsic time-scales. Because of the independence of the unforced response of the pre-shock turbulence, the analysis of this phenomenon is impossible within the present framework, and beyond the scope of the present work.

For weakly unstable detonations the unsteadiness of the mean structure can be separated form the perturbation dynamics by assuming that its time scale is much larger than the gas residence time. This assumption leads to the commonly used WKB (Wentzel-Kramers-Brillouin) description of the problem: a slow varying mean field and a fast convected perturbation, (see Huerre ${ }^{11}$ for applications of the WKB analysis to spatially developing flows). On the other hand, the intrinsic fluctuation is expected to be smaller than the forced response because of the resonant amplification of the latter. Thus, we conclude that, for weakly unstable structures, the detonation-turbulence interaction is dominated by the resonant part of the response evaluated with respect to a quasi-steady mean solution. Such mean solution is approximated as the $1 \mathrm{D}$ detonation structure.

The natural frequencies of a detonation wave provide information about maximally amplified pre-shock turbulent components. Detonation structure parameters and pre-shock wave numbers supporting such nonforced oscillating solutions are summarized in Fig. 7 for a $E=30, \gamma=1.2$, and $f=1.2$ detonation structure. Figure 7(a) shows on the ordinate the pre-shock longitudinal natural wave numbers against the heat release on the abscissa. The number of characteristic solutions increases with an increase in heat release. The minimum longitudinal wave number supporting a natural solution is $\hat{k}_{1} / L^{*}=0.303$, at $Q \approx 1.28$. Such value gives an indication on the maximum longitudinal turbulence length scales likely to interact with the detonation structure. The range of characteristic wave numbers supported by a structure, $\left[\min \left(\hat{k}_{1} / L^{*}\right), \max \left(\hat{k}_{1} / L^{*}\right)\right]$, increases with the activation energy according to Fig. $7(\mathrm{c})$, so that we infer that small scale turbulence will interact at the resonant level with high activation energy detonations. Figure 7(b) shows the radial wave number. Eigenvalues disappear from the map when the radial wave number approaches zero, and Fig. $7(\mathrm{~d})$ demonstrates that this condition occurs for pre-shock angles approaching $\pi / 2$, thus for non-zero longitudinal numbers. For $\theta_{0}=\pi / 2$ the pre-shock and post-shock perturbations are aligned with the longitudinal axis. Figure 7 (c) shows that for low heat release the majority of the eigensolutions are super-critical, but when $Q$ is increased the solutions shift towards the sub-critical half plane $\theta_{0} / \theta_{c r}<1$. Eigenvalues appear on the map in pairs and they separate as the heat release is increased from the inception point. One increases in radial wave number and decreases in pre-shock angle, while the other follows the opposite path.

Figure 8 shows the natural solution map for a $E=30, \gamma=1.2$ and $f=2.0$ detonation structure. Conclusions similar to those drawn for Fig. 7 apply to the increased overdrive case. The most evident effect of an increase in overdrive is a reduction of natural frequencies and a shift of the natural wave numbers towards zero. At $Q=2$, the range of resonant wave numbers for $f=2$ is significantly smaller than for $f=1.2$. Unstable solutions occur for a lower value of the heat release and the ratio $\theta_{0} / \theta_{c r}$ is reduced.

Figure 9 shows the one-dimensional power spectra for $E=30, \gamma=1.2, f=1.2, L^{*}=1$ and four values of the heat release in the range $Q \in[0.755,2]$. Since the $\hat{k}_{1, i}^{*}$ eigenvalues are singularities, the spectrum is evaluated over the collection of intervals

$$
\bigcup_{i=1}^{n^{*}+1}\left[\hat{k}_{1, i-1}^{*}+1 \times 10^{-3}, \hat{k}_{1, i}^{*}-1 \times 10^{-3}\right],
$$

where $n^{*}$ is the number of natural eigenvalues for a given detonation structure, $\hat{k}_{1,0}^{*}=0$ and $\hat{k}_{1, n^{*}+1}^{*}=10$. Large-scale (low wave number) amplification is stronger for values of the heat release close to the stability limit, i.e., $Q=0.756$ solid line. This result is expected based on the disappearance of the minimum natural wave number for $Q>1.28$. The presence of characteristic solutions with radial wave number close to zero $\left(\theta_{0} \rightarrow \pi / 2\right)$ has a weak effect on the spectrum. The dashed line in Figs. 9(a) and 9(c) show that a local minimum in the acoustic spectrum is present for heat releases close to the previously discussed value $Q=1.28$. Results for $Q=2$ (dotted line) show that the presence of eigenvalues very close in wave number 


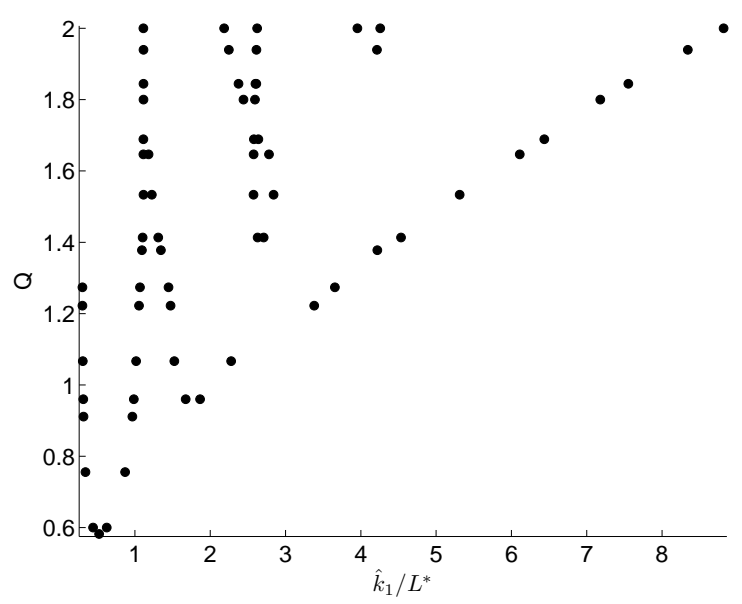

(a)

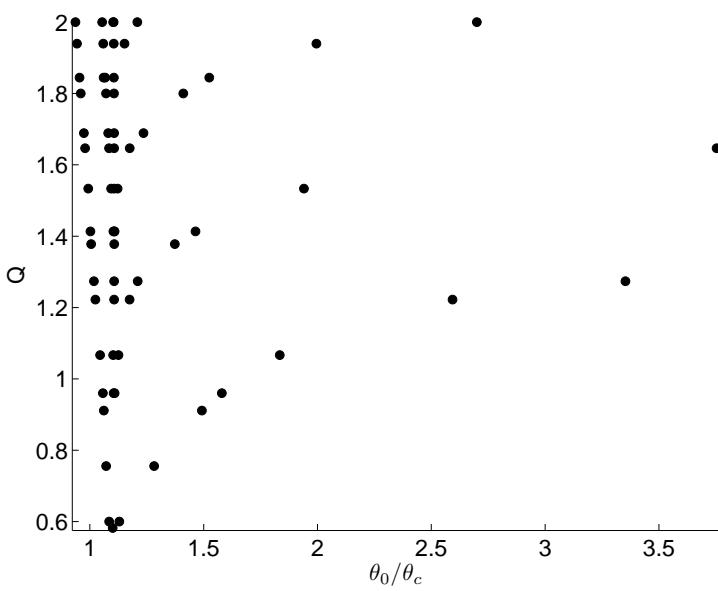

(c)

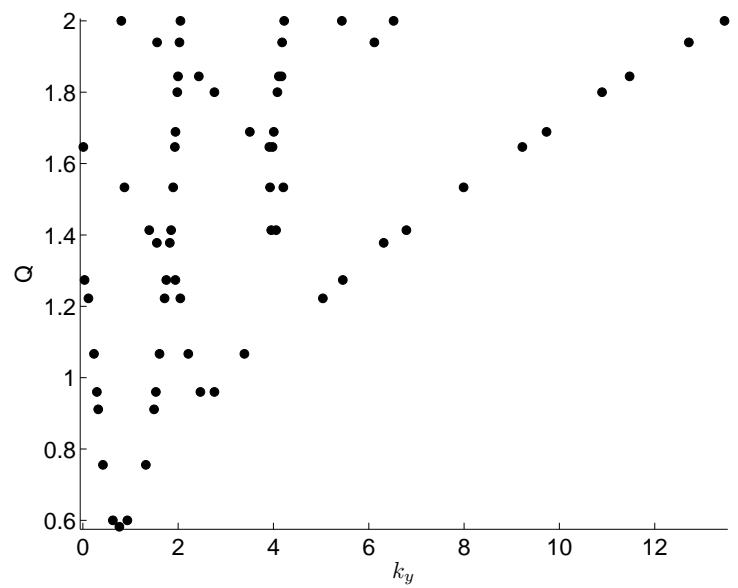

(b)

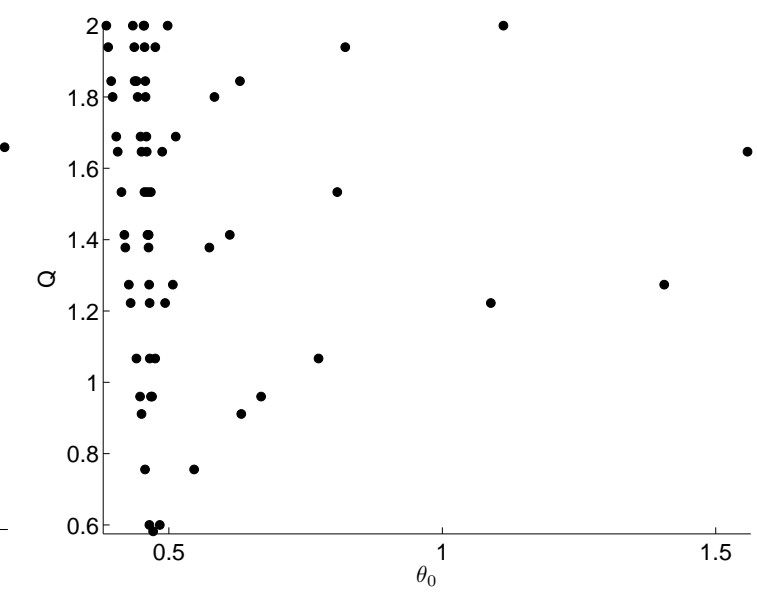

(d)

Figure 7. Eigenvalue map of natural frequencies for a $E=30, \gamma=1.2$ and $f=1.2$ detonation structure. The ordinate in all plots is the heat release. 


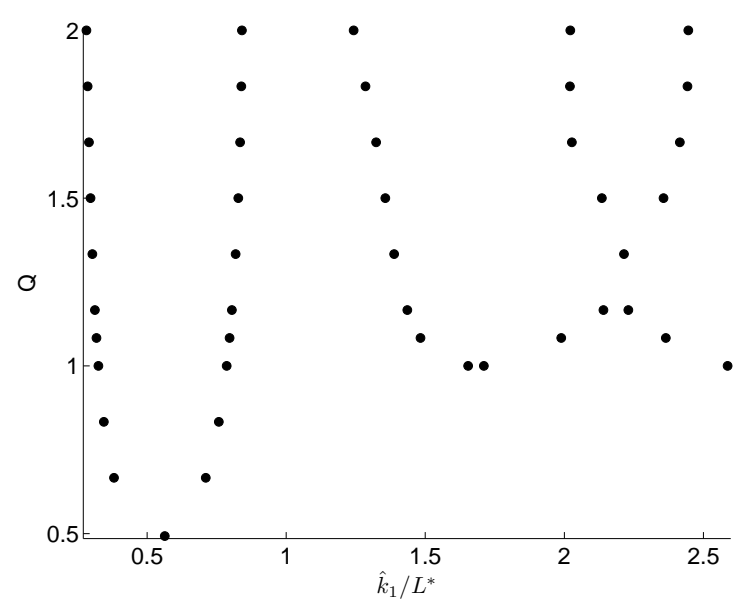

(a)

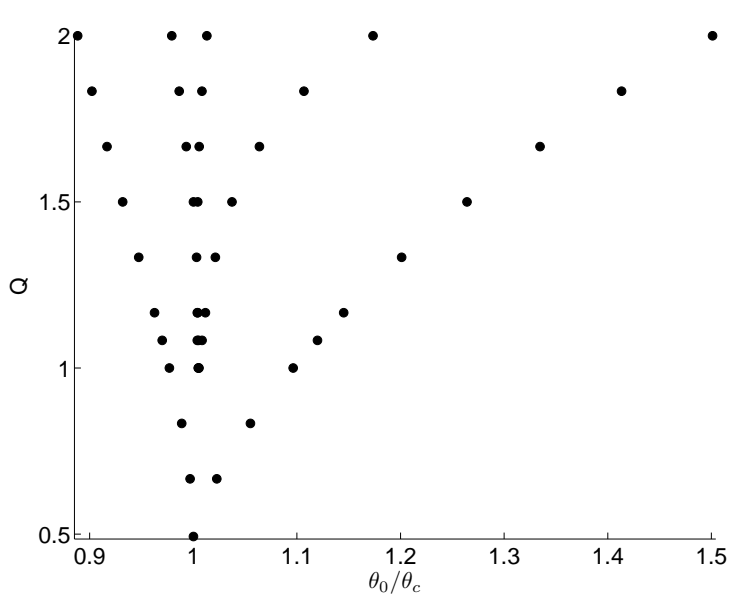

(c)

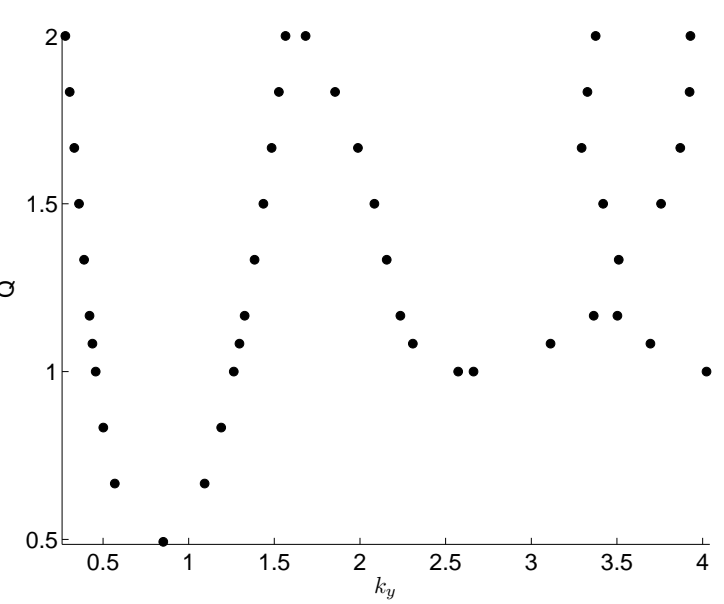

(b)

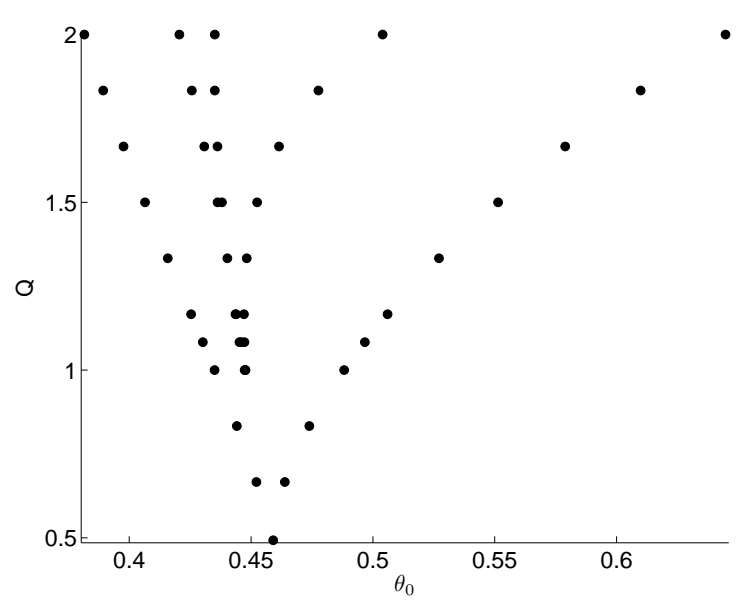

(d)

Figure 8. Eigenvalue map of natural frequencies for a $E=30, \gamma=1.2$ and $f=2.0$ detonation structure. The ordinate in all plots is the heat release.

16 of 20 
is particularly effective in increasing both the entropy and the acoustic far-fields. Therefore, maximum amplification is expected for conditions close to the inception point of an eigenvalue pair.

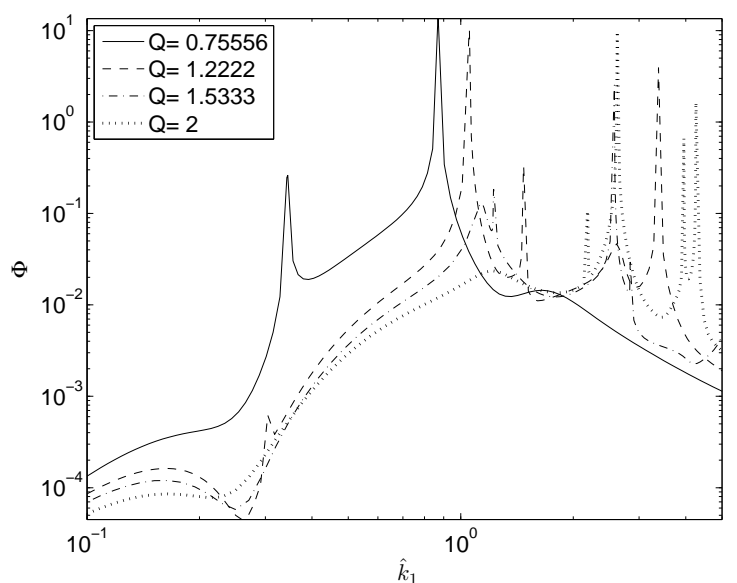

(a) Pressure Acoustic Contribution

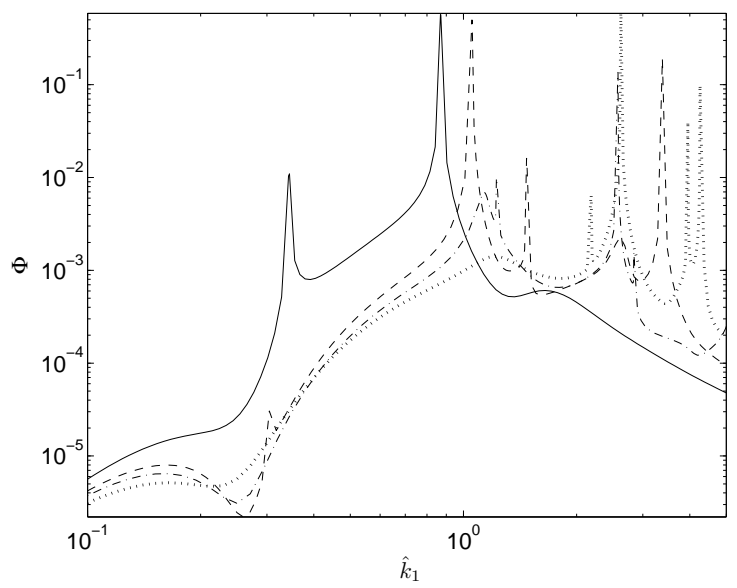

(c) Temperature Acoustic Contribution

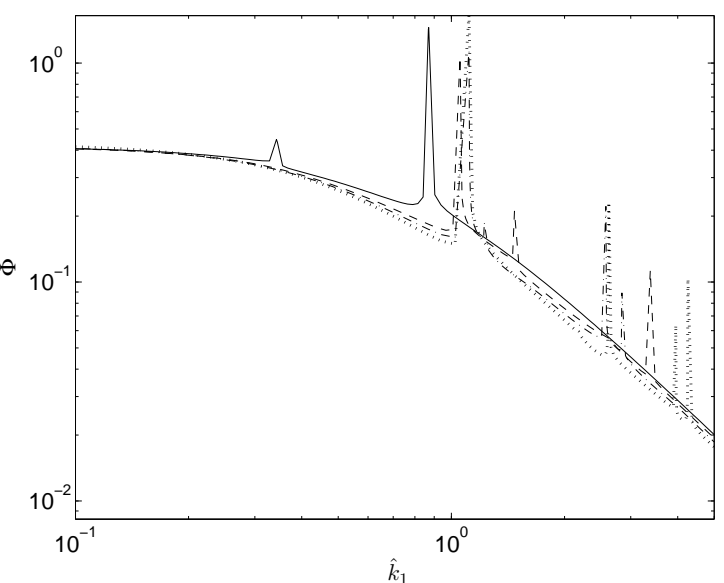

(b) Velocity Entropy Contribution

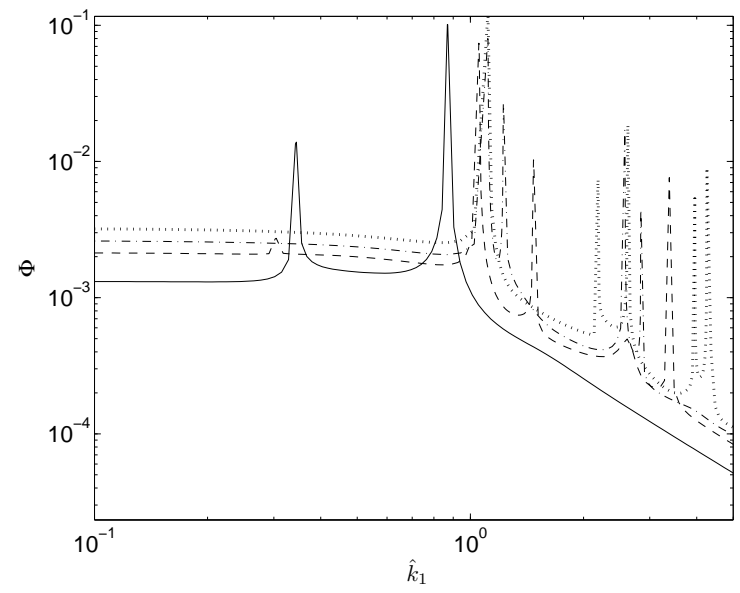

(d) Temperature Entropy Contribution

Figure 9. One-dimensional power spectra for $E=30, \gamma=1.2, f=1.2$ and four values of the heat release parameter. The legend shown only in panel (a) applies to the four panels.

\section{VIII.D. $\quad L^{*}$ Effect on a Detonation Close to the Stability Limit}

In this section, we analyze the effect of the ratio between turbulence longitudinal length scale and detonation half reaction length $\left(L^{*}\right)$ on the one-dimensional spectra of a $Q=2, E=10, \gamma=1.2, f=1.2$ detonation. One-dimensional spectra are plotted against the longitudinal wave number in Fig. 10 for $L^{*}=1,5,10$. The shift of the peak frequencies to the left with an increase in $L^{*}$ is expected based on the notion that the homogeneous system is not affected by external forcing and thus independent of such a parameter.

Entropy and acoustic contributions to the post-shock temperature power spectra are shown in Fig. 11 for three value of $L^{*}$. Both acoustic and entropy contributions are strongly affected by the detonation structure for the 3 values of $L^{*}$ considered. Therefore, turbulence with a longitudinal scale up to ten times the reaction 


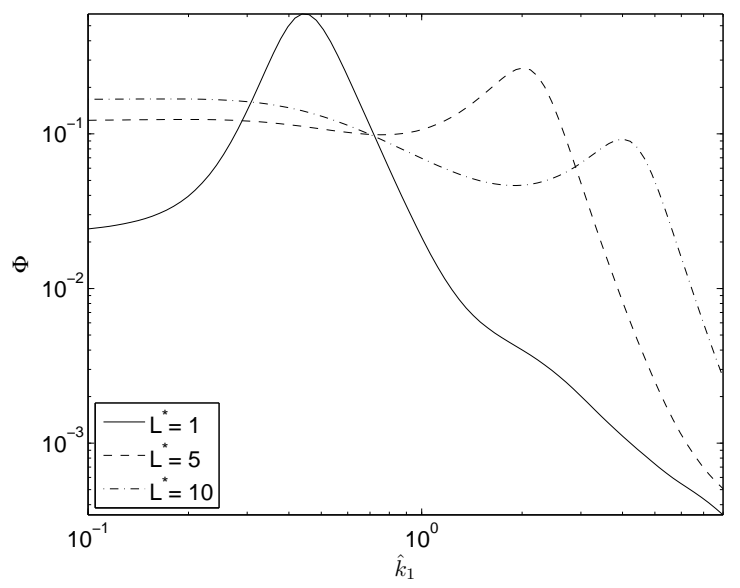

(a) Pressure at $x=1$

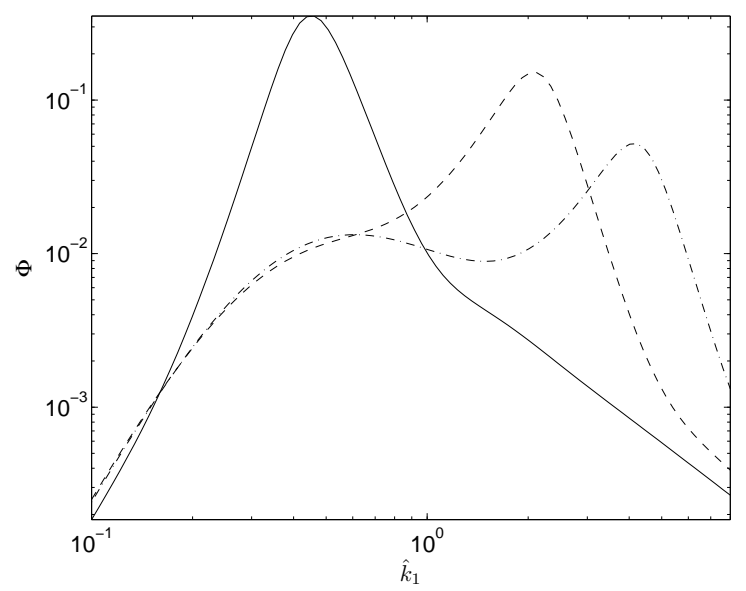

(c) Pressure Acoustic Contribution

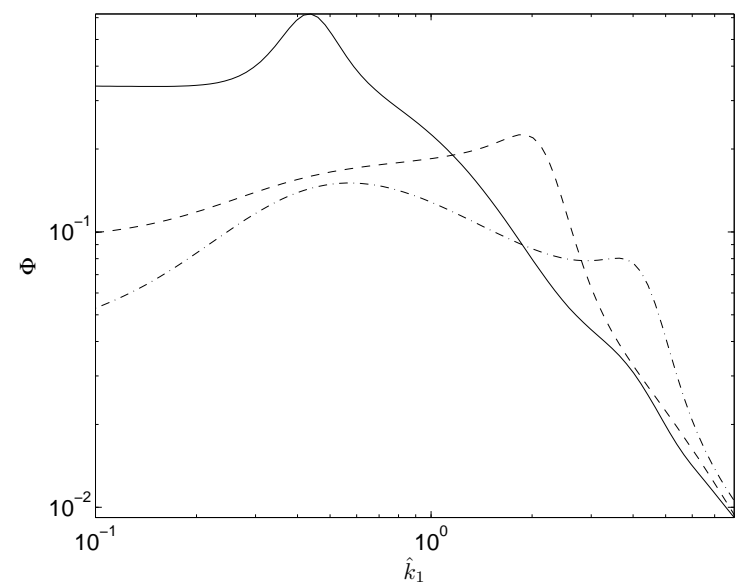

(b) Velocity at $x=1$

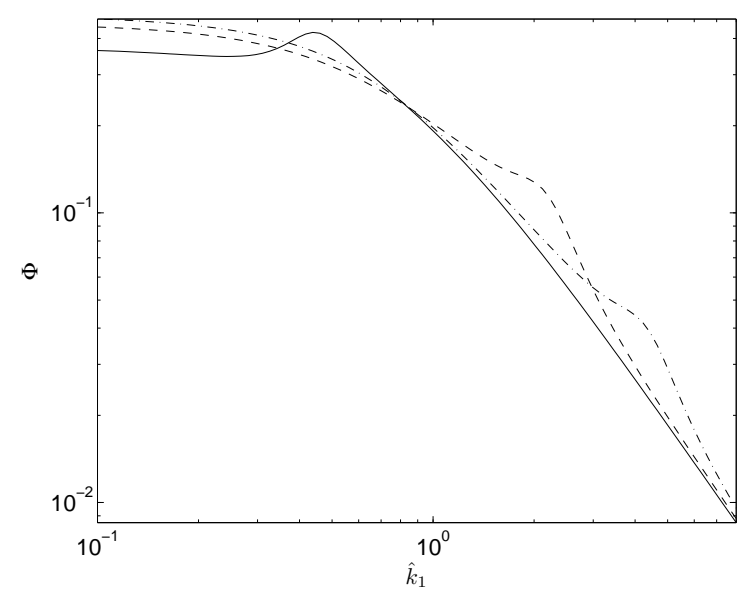

(d) Velocity Entropy Contribution

Figure 10. Variation of the velocity and pressure power spectrum with $L^{*}$ for a $Q=2, \gamma=1.2, f=1.2$, and $E=10$ structure. The legend shown only in panel (a) applies to the four panels. 


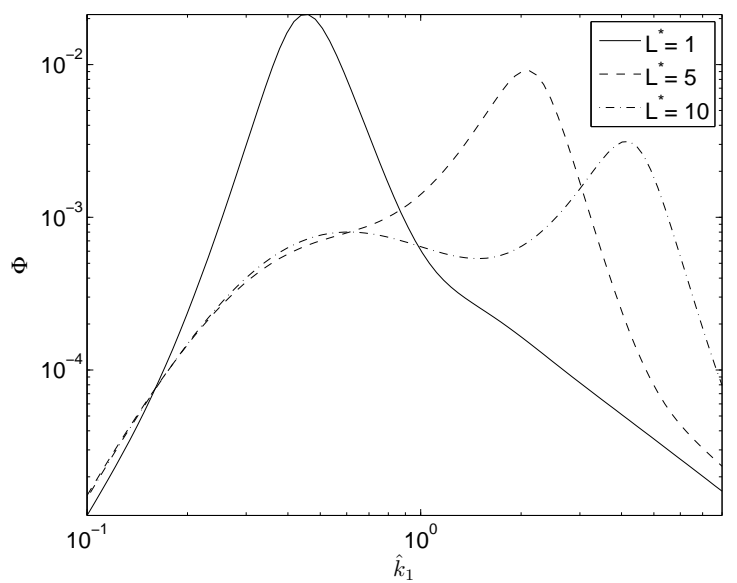

(a) Temperature Acoustic Contribution

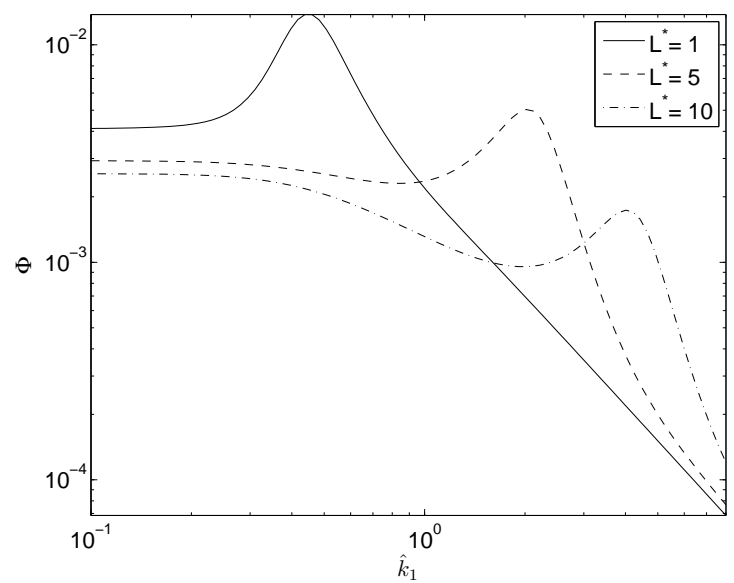

(b) Temperature Entropy Contribution

Figure 11. Variation of the temperature power spectrum with $L^{*}$ for a $Q=2, \gamma=1.2, f=1.2$, and $E=10$ structure.

half length interacts with the detonation structure. As $L^{*}$ increases, finer turbulent structures are excited by the resonance. The effect of an increase in $L^{*}$ on the coarse scale entropy far-field, $\hat{k}_{1} \rightarrow 0$, is to increase the longitudinal velocity and to decrease the temperature.

\section{Conclusions}

We analyze the influence of structural scales on the amplification of turbulence passing through a detonation wave. A set of characteristic scales is identified based on the eigenvalues of the Euler linearized PDE in the induction region. Two-dimensionally unstable detonations possess a set of real characteristic wave numbers or natural wave numbers, at which the system oscillates freely. The transfer functions are singular at the corresponding longitudinal wave numbers, and resonance produces unbounded amplification in the post-shock spectra. The size of the set of natural wave numbers and the range of frequencies increase with the heat release, at a given activation energy. Thus, high activation energy detonations interact with a wider range of fine turbulent scales. On the other hand, minimum natural wave numbers occurs for low value of the heat release, so large-scale amplification is expected for conditions close to the stability limit. For low values of the heat release, just above the stability boundary, the characteristic solutions are super-critical; an increase in heat release changes part of the eigensolutions into sub-critical. Sub-critical solutions do not excite the acoustic far-field by resonance. The strongest resonance effect on power spectra occurs when two natural wave numbers are close, which occurs at the point of inception of an instability mode.

Two-dimensionally stable detonations do not have any purely real eigenvalues, and characteristic (nonnatural) wave numbers are associated with eigenvalues with minimum imaginary part. The effect of the characteristic scales is to produce local maxima in the post-shock spectra. For all cases studied, the characteristic frequencies have a stronger influence on the acoustic field than on the entropy field behind the wave. A decrease in activation energy form the value on the stability surface strongly weakens the resonant amplification. We conclude that activation energy plays a strong role on turbulence amplification.

Significant information on weakly stable waves can be gained analyzing natural eigensolutions on the stability boundary. We find that an increase in overdrive decreases the value of heat release for a given activation energy, an observation that challenges the notion that increase in overdrive leads to stability. The ratio between pre-shock wave angle and its critical value is close to unity for $f \leq 2$ and $E \leq 30$. An increase in activation energy or a decrease in overdrive pushes natural solution towards the super-critical half plane $\theta_{0} \geq \theta_{c r}$. We conclude that increasing the overdrive acts to attenuate resonant acoustic amplification. On 
the stability boundary, the natural wave numbers increase with the activation energy and decrease with the overdrive for sub-critical solutions. Therefore, structures with larger overdrive support resonant amplification of coarser turbulent scales. For detonation conditions on the stability boundary, a larger overdrive yields a weaker resonant peak in both temperature and longitudinal velocity spectra.

Finally, the ratio of longitudinal turbulence length to reaction half distance $L^{*}$ acts to shift characteristic frequencies. For a weakly stable detonation, we find that ratios up to ten yield strong resonant behavior. Increasing $L^{*}$ has opposite effects on the temperature and velocity entropy far-field of large turbulent scales. It enhances the longitudinal velocity but it diminishes the temperature entropy contribution.

Future work will focus on the non-linear analysis of the interaction and on the effect of viscosity near resonance conditions. The effect of complex chemistry on the interaction is another problem we plan to investigate.

\section{References}

${ }^{1}$ H.S. Ribner, Spectra of Noise and Amplified Turbulence Emanating from Shock-Turbulence Interaction, AIAA J. 25(3):436-442, (1987).

${ }^{2}$ T.L. Jackson, A.K. Kapila and M.Y. Hussaini, Convection of a pattern of vorticity through a reacting shock wave, Phys. of Fluids, 2(7):1260-1268, (1990).

${ }^{3}$ T.L. Jackson, M.Y. Hussaini and H.S. Ribner, Interaction of turbulence with a detonation wave, Phys. of Fluids, 5(3):745$749,(1993)$.

${ }^{4}$ J.J. Erpenbeck, Stability of Idealized one-reaction Detonations, The Physics of Fluids, 7(5):684-696, (1964).

${ }^{5}$ M. Short and D.S. Stewart, Cellular detonation stability. Part 1. A normal mode linear analysis, J. Fluid Mech., 368:229262, (1998).

${ }^{6}$ J.O. Hinze, Turbulence, McGraw-Hill, p. 247, (1975).

${ }^{7}$ J.E. Moyal, The spectra of turbulence in a compressible fluid; eddy turbulence and random noise, Proc. Cam. Phil. Soc., 48(2):329-344, (1952).

${ }^{8}$ H.S. Ribner, Convection of a pattern of vorticity through a shock wave, NACA Report 1164, (1954).

${ }^{9}$ J. Dongarra, B. Straughan and D. Walker, Chebyshev tau-QZ algorithm methods for calculating spectra of hydrodynamic stability problems, Applied Numerical Mathematics 22:399-434, (1996).

${ }^{10}$ C. Moler and G. Stewart, An algorithm for generalized matrix eigenproblems, SIAM J. Numer. Anal. 10 241-256, (1973).

${ }^{11}$ P. Huerre, Local and global instabilities in spatially developing flows, Annu. Rev. Fluid Mech. 22:473-537 (1990). 